\title{
A Simple Test on Structural Change in Long-Memory Time Series*
}

\author{
Kai Wenger $^{a} \quad$ Christian Leschinski $^{a} \quad$ Philipp Sibbertsen $^{a}$ \\ ${ }^{a}$ Leibniz University Hannover
}

April 13, 2017

\begin{abstract}
We propose a simple test on structural change in long-range dependent time series. It is based on the idea that the test statistic of the standard CUSUM test retains its asymptotic distribution if it is applied to fractionally differenced data. We prove that our approach is asymptotically valid if the memory is estimated consistently under the null hypothesis. Therefore, the well-known CUSUM test can be used on the differenced data without any further modification. In a simulation study, we compare our test with a CUSUM test on structural change that is specifically constructed for long-memory time series and show that our approach performs well.
\end{abstract}

Key words: Fractional Integration $\cdot$ Structural Breaks $\cdot$ Long Memory.

JEL classification: $\mathrm{C} 12, \mathrm{C} 22$

\section{Introduction}

Testing for structural stability in linear time series regression is a widely studied field in the financial and economic literature since parameter instability invalidates estimation and forecasts (see Perron et al. (2006), Andreou and Ghysels (2009), among others).

One of the most prominent change-in-mean tests is the CUSUM test that was originally proposed by Brown, Durbin, and Evans (1975). Here, we focus on the version of Krämer, Ploberger, and Alt (1988) that is based on OLS residuals and tends to have better power properties than the original version based on recursive residuals. However, our results also apply to the recursive version of the test.

The standard CUSUM test has the shortcoming that it is developed for independent or serially correlated series, but not for long-range dependent data. Long-memory time series and series with shifts in mean are easily confused since they can show similar characteristics such as hyperbolically decaying autocorrelations or a pole in the periodogram at Fourier frequencies close to zero (see for example Diebold and Inoue (2001) or Granger and Hyung (2004)). Therefore,

*Financial support of the Deutsche Forschungsgesellschaft (DFG) is gratefully acknowledged. 
several papers show that standard CUSUM tests are invalid in presence of long memory. Wright (1998) and Krämer and Sibbertsen (2002) show that the limiting distribution of the CUSUM test under long-memory is different to the short-memory case. This results in the fact that the standard test always rejects asymptotically when $d>0$. The normalization factor of the CUSUM statistic needs to be larger to account for the slow convergence of the highly persistent long-memory series. Furthermore, the limiting distribution in the short-memory case is based on independent increments, whereas the increments in the long-memory limit distribution are not independent.

A solution is to use another normalization factor and critical values from a fractional Brownian bridge such that the statistic converges to a well defined process and has correct critical values. For Gaussian processes this is done by Horváth and Kokoszka (1997) and extended by Wang (2008) to a wider range of linear processes. However, the modified tests converge to nonstandard limiting distributions that depend on $d$.

This paper proposes a different approach as an intuitive alternative particularly for practitioners. In a two-step procedure we first estimate the long-memory parameter and then perform the CUSUM test for structural change on the $\widehat{d}$ times differenced data. The local Whittle estimator is used for estimating $d$ which is consistent under the null hypothesis of no structural change.

We show theoretically that the well known CUSUM test can be used in long-memory times series after fractional differentiation of the data. Hence, at least asymptotically, there is no need to use the modified versions of the standard tests. This has the advantage that the standard CUSUM test is implemented in most common software packages for statistical analyses.

The rest of the paper is organized as follows. Section 2 describes the model along with the structural change tests considered here. Section 3 provides and discusses our new test based on differenced data. In Section 4 a Monte-Carlo simulation is conducted and Section 5 concludes.

\section{CUSUM type tests}

We consider a signal-plus-noise model ${ }^{1}$ where the observations $\left(y_{t}\right)_{t \geq 1}$ are generated by the stochastic process

$$
y_{t}=\mu_{t}+\epsilon_{t}
$$

Here, the regression means $\left(\mu_{t}\right)_{t \geq 1}$ are assumed to be deterministic and fulfill $\left|\mu_{t}\right|<\infty$, for all $t=1, \ldots, T$. For the error term we assume that $\epsilon_{t}=\Delta^{-d} v_{t}$, where $v_{t}$ is a mean zero martingale difference sequence with finite variance, $|d|<1 / 2$, and $\Delta^{d}=(1-L)^{d}$ is the fractional differencing operator defined as

$$
(1-L)^{d}=\sum_{k=0}^{\infty} \pi_{k}(d) L^{k}=\sum_{k=0}^{\infty} \frac{\Gamma(k-d)}{\Gamma(-d) \Gamma(k+1)} L^{k}
$$

That means we consider a process with a possibly time varying mean and stationary fractionally integrated errors $\epsilon_{t}$.

\footnotetext{
${ }^{1}$ One could also assume a standard regression model with $\mu_{t}=\beta_{t}^{\prime} x_{t}$ and test whether $\beta_{t}=\beta$.
} 
Given a specific time series $y_{1}, \ldots, y_{T}$ our interest lies in testing the null hypothesis of a constant unconditional mean

$$
H_{0}: \mu_{1}=\ldots=\mu_{T}=\mu
$$

against the alternative of a shift in mean

$$
H_{1}: \mu_{t} \neq \mu_{s} \quad \text { for some } \quad 1<t \neq s<T \text {. }
$$

The CUSUM test statistic under long memory (CUSUM-LM) proposed by Horváth and Kokoszka (1997) and Wang (2008) is based on

$$
S_{T}(\tau, \widehat{d})=\frac{1}{\widehat{\sigma}_{c} T^{1 / 2+\widehat{d}}}\left(\sum_{t=1}^{\lfloor\tau T\rfloor} \widehat{\epsilon}_{t}-\frac{\lfloor\tau T\rfloor}{T} \sum_{t=1}^{T} \widehat{\epsilon}_{t}\right), \quad \tau \in[0,1],
$$

where $\lfloor\cdot\rfloor$ denotes the integer part of its arguments, $\widehat{\sigma}_{c}$ and $\widehat{d}$ are consistent estimators of the long run variance and the memory parameter and $\widehat{\epsilon}_{t}$ is an estimate of the residuals from (1). The test statistic is given by

$$
Q_{T}=\sup _{0 \leq \tau \leq 1}\left|S_{T}(\tau, \widehat{d})\right|
$$

For $T \rightarrow \infty$ it converges in distribution to the supremum of a fractional Brownian bridge

$$
Q_{T} \stackrel{d}{\rightarrow} \sup _{0 \leq \tau \leq 1}\left|B_{d}(t)-t B_{d}(1)\right|
$$

where $B_{d}(t)$ is a fractional Brownian motion and $\stackrel{d}{\rightarrow}$ denotes convergence in distribution.

The statistic also nests the standard CUSUM test for short-memory time series data when $d=0$ and $\widehat{\sigma}_{c}$ is an autocorrelation consistent (HAC) long run variance estimator (cf. for example Newey and West (1987), Andrews (1991)). In this case the statistic converges to the supremum of a standard Brownian bridge.

\section{CUSUM test after fractional differentiation}

Instead of using the modified CUSUM-LM test, we suggest to use the standard CUSUM test on the fractionally differenced series

$$
y_{t}^{*}(\widehat{d})=\Delta^{\widehat{d}} y_{t}=\Delta^{\widehat{d}} \mu_{t}+\Delta^{\widehat{d}} \epsilon_{t}=\mu_{t}^{*}+v_{t}^{*}
$$

Now, denote the partial sum statistic from (5) calculated from $\widehat{v}_{t}^{*}$ and under the assumption that $d=0$, by $S_{T}^{*}(\tau, 0)$. The corresponding CUSUM test statistic is denoted by $Q_{T}^{*}$.

Both approaches, the CUSUM-LM test as well as ours, require a consistent estimate of $d$ under $H_{0}$. Our test requires that for $T \rightarrow \infty$ the differenced series $y_{t}^{*}(\widehat{d})$ is $I(0)$ and the CUSUM-LM test needs an estimator of $d$ directly in the denominator of the test statistic, and indirectly to obtain the correct critical values. This estimate is usually obtained using the local Whittle 
estimator of Künsch (1987) and Robinson (1995).

By using fractional differences we implicitly assume that $y_{t}=\mu_{t}$ and $\epsilon_{t}=0$, for $t \leq 0$, which means that the process was equal to its mean before the beginning of the sample period. This corresponds to a fractionally integrated process of type II. Fractionally integrated processes of type I, on the other hand, assume that $\epsilon_{t}$ has an infinite past. For a detailed discussion of type I and type II fractional Brownian motions see Marinucci and Robinson (1999).

Different from our differencing approach, the CUSUM-LM test usually assumes a type-I process. However, for $0 \leq d<1 / 2$, type I and type II processes are asymptotically equivalent.

The following theorem provides the limiting distribution of the test statistic

Theorem 1. Suppose that $\widehat{d}-d_{0}=o_{P}\left(T^{-\eta}\right)$, for some arbitrary small $\eta>0$. Then under $H_{0}$ : $Q_{T}^{*} \stackrel{d}{\rightarrow} \sup _{0 \leq \tau \leq 1}\left|B_{0}(t)-t B_{0}(1)\right|$.

Proof. To prove the theorem, we show that the partial sum statistic $S_{T}^{*}(\tau, 0)$ calculated from the differenced error process converges in distribution to $S_{T}^{v}(\tau, 0)$ - the statistic based on the true innovation sequence $v_{t}$. Denote $\delta=d-\widehat{d}$, then under $H_{0}$ with $\epsilon_{t}=\Delta^{-d} v_{t}$ and $v_{t}^{*}=\Delta^{\widehat{d}} \epsilon_{t}$ from (1) and (7), we have

$$
\begin{aligned}
v_{t}^{*} & =\Delta^{-\delta} v_{t}=v_{t}+\sum_{k=1}^{\infty} \theta_{k}(\delta) v_{t-k}, \\
\text { where } \quad \theta_{k}(\delta) & =\frac{\Gamma(k+\delta)}{\Gamma(\delta) \Gamma(k+1)} .
\end{aligned}
$$

Therefore, the difference between the fractionally differenced error series $v_{t}^{*}$ and the original innovation sequence $v_{t}$ is given by

$$
\gamma_{t}=v_{t}^{*}-v_{t}=\sum_{k=1}^{\infty} \theta_{k}(\delta) v_{t-k}
$$

By a first order Taylor approximation of the $\theta_{k}(\delta)$ around $\delta=0$, we have

$$
\gamma_{t}=O_{p}(\delta) \tilde{\gamma}_{t}=O_{p}(\delta) \sum_{k=1}^{\infty} \frac{v_{t-k}}{k},
$$

since

$$
\frac{\partial \theta_{k}(\delta)}{\partial \delta}=\frac{\psi_{0}(\delta+k) \Gamma(\delta+k)}{\Gamma(k+1) \Gamma(\delta)}-\frac{\psi_{0}(\delta)}{\Gamma(\delta)} \frac{\Gamma(k+\delta)}{\Gamma(k+1)}
$$

$\Gamma(0)=\infty, \Gamma(k) / \Gamma(k+1)=1 / k$, and $\psi_{0}(0) / \Gamma(0)=-1$, where $\psi_{0}(\delta)$ denotes the Digamma function. Denote

$$
k^{*}=\min \left\{k: \frac{1}{k}<\frac{k^{\eta-1}}{\Gamma(\eta)}\right\}
$$


then we can rewrite $\tilde{\gamma}_{t}$ from (9) as

$$
\tilde{\gamma}_{t}=\sum_{k=1}^{k^{*}-1} \frac{v_{t-k}}{k}+\sum_{k=k^{*}}^{\infty} \frac{v_{t-k}}{k}=\tilde{\gamma}_{t}^{(1)}+\tilde{\gamma}_{t}^{(2)}
$$

such that from (8) and $\delta=o_{p}\left(T^{-\eta}\right)$, we have

$$
S_{T}^{*}(\tau, 0)-S_{T}^{v}(\tau, 0)=\frac{o_{p}\left(T^{-\eta}\right)}{\widehat{\sigma}_{c} T^{1 / 2}}\left(\sum_{t=1}^{\lfloor\tau T\rfloor} \tilde{\gamma}_{t}^{(1)}+\sum_{t=1}^{\lfloor\tau T\rfloor} \tilde{\gamma}_{t}^{(2)}-\frac{\lfloor\tau T\rfloor}{T}\left(\sum_{t=1}^{T} \tilde{\gamma}_{t}^{(1)}+\sum_{t=1}^{T} \tilde{\gamma}_{t}^{(2)}\right)\right) .
$$

Obviously, $\tilde{\gamma}_{t}^{(1)}$ is short memory, so that the partial sum grows with rate $O_{p}(\sqrt{T})$ and $o_{P}\left(T^{-\eta-1 / 2}\right)$ $\sum_{t=1}^{\lfloor\tau T\rfloor} \gamma_{t}^{(1)}=o_{p}\left(T^{-\eta}\right)$. Furthermore, we have

$$
\tilde{\gamma}_{t}^{(2)}=\sum_{k=1}^{\infty} a_{k} v_{t-k}
$$

where $a_{k}=k^{-1}$ for $k \geq k^{*}$ and zero otherwise. Hence, by (10), we have $a_{k} \leq \frac{k^{\eta-1}}{\Gamma(\eta)}$ for all $k=1,2, \ldots$, so that the growth rate of the partial sum of a long-memory process with parameter $\eta$ is an upper bound for that of $\tilde{\gamma}_{t}^{(2)}$ :

$$
\sum_{t=1}^{\lfloor\tau T\rfloor} \tilde{\gamma}_{t}^{(2)} \leq O_{p}\left(T^{1 / 2+\eta}\right)
$$

Therefore, $o_{p}\left(T^{-\eta}\right) T^{-1 / 2} \sum_{t=1}^{\lfloor\tau T\rfloor} \gamma_{t}^{(2)} \leq O_{p}\left(T^{\eta}\right) o_{p}\left(T^{-\eta}\right)=o_{p}(1)$. Together with the previous results this implies $S_{T}^{*}(\tau)-S_{T}^{v}(\tau)=o_{p}(1)$ and consequently

$$
Q_{T}^{*} \stackrel{d}{\rightarrow} Q_{T}^{v}
$$

The convergence of the supremum follows from the continuous mapping theorem.

Theorem 1 requires a consistent estimate of $d$ that satisfies a minimal rate assumption. This is fulfilled by standard semiparametric estimators such as the local Whittle estimator. Under this condition, the standard CUSUM test applied to the differenced data converges to the usual statistic.

Remark 1. In practice both $S_{T}(\tau, \widehat{d})$ and $S_{T}^{*}(\tau, 0)$ are based on estimates $\widehat{\epsilon}_{t}$ and $\widehat{\epsilon}_{t}^{*}$ of the error processes that are obtained by de-meaning the observed series $y_{t}$ and $y_{t}^{*}$. To simplify the exposition we did not consider the effect of estimation errors introduced by de-meaning in the proof of Theorem (1). Nevertheless, the extension of the results is straightforward.

\section{Simulation results}

The finite sample size and power properties of our test are examined in a Monte Carlo simulation study and compared with the CUSUM-LM test. We simulate fractionally integrated white noise and choose $d \in\{0 ; 0.2 ; 0.4\}$ and sample sizes $T \in\{250 ; 500 ; 1000 ; 2500\}$. We apply the local 


\begin{tabular}{|c|c|c|c|c|c|c|c|c|c|c|c|c|}
\hline \multirow[t]{4}{*}{$\mathrm{n} / \mathrm{b}$} & \multicolumn{2}{|c|}{0.70} & \multicolumn{2}{|c|}{0.75} & \multicolumn{2}{|c|}{0.80} & \multicolumn{2}{|c|}{0.70} & \multicolumn{2}{|c|}{0.75} & \multicolumn{2}{|c|}{0.80} \\
\hline & \multicolumn{6}{|c|}{ Size } & \multicolumn{6}{|c|}{ Power } \\
\hline & \multicolumn{12}{|c|}{$d=0$} \\
\hline & I & II & I & II & I & II & I & II & $\mathrm{I}$ & II & $\mathrm{I}$ & II \\
\hline 250 & 0.017 & 0.017 & 0.026 & 0.024 & 0.028 & 0.026 & 0.884 & 0.983 & 0.985 & 0.997 & 0.998 & 0.999 \\
\hline 500 & 0.022 & 0.024 & 0.027 & 0.030 & 0.031 & 0.032 & 0.989 & 1.000 & 1.000 & 1.000 & 1.000 & 1.000 \\
\hline 1000 & 0.029 & 0.033 & 0.033 & 0.037 & 0.036 & 0.039 & 1.000 & 1.000 & 1.000 & 1.000 & 1.000 & 1.000 \\
\hline \multirow[t]{2}{*}{2500} & 0.036 & 0.039 & 0.039 & 0.045 & 0.039 & 0.045 & 1.000 & 1.000 & 1.000 & 1.000 & 1.000 & 1.000 \\
\hline & \multicolumn{12}{|c|}{$\mathrm{d}=0.2$} \\
\hline 250 & 0.014 & 0.016 & 0.021 & 0.022 & 0.028 & 0.025 & 0.344 & 0.488 & 0.517 & 0.595 & 0.652 & 0.701 \\
\hline 500 & 0.022 & 0.025 & 0.024 & 0.028 & 0.032 & 0.031 & 0.632 & 0.834 & 0.828 & 0.912 & 0.919 & 0.947 \\
\hline 1000 & 0.026 & 0.029 & 0.030 & 0.034 & 0.038 & 0.042 & 0.897 & 0.986 & 0.982 & 0.997 & 0.995 & 0.998 \\
\hline \multirow[t]{2}{*}{2500} & 0.030 & 0.032 & 0.038 & 0.041 & 0.042 & 0.043 & 0.998 & 1.000 & 1.000 & 1.000 & 1.000 & 1.000 \\
\hline & \multicolumn{12}{|c|}{$\mathrm{d}=0.4$} \\
\hline 250 & 0.011 & 0.013 & 0.016 & 0.019 & 0.026 & 0.023 & 0.106 & 0.161 & 0.128 & 0.185 & 0.168 & 0.212 \\
\hline 500 & 0.014 & 0.018 & 0.018 & 0.022 & 0.029 & 0.027 & 0.138 & 0.240 & 0.169 & 0.260 & 0.237 & 0.307 \\
\hline 1000 & 0.016 & 0.022 & 0.024 & 0.028 & 0.036 & 0.036 & 0.178 & 0.323 & 0.249 & 0.370 & 0.328 & 0.411 \\
\hline 2500 & 0.025 & 0.030 & 0.026 & 0.031 & 0.042 & 0.041 & 0.295 & 0.471 & 0.397 & 0.516 & 0.485 & 0.572 \\
\hline
\end{tabular}

Table 1: Size and power results of the CUSUM test on $\widehat{d}$ times differenced data (I) and the CUSUM-LM type test (II) applied on fractionally integrated white noise for different bandwidths $m=\left\lfloor T^{b}\right\rfloor$ and memory parameters $d$.

Whittle estimator to estimate $d$ using bandwidths $m=\left\lfloor T^{b}\right\rfloor$ with $b \in\{0.7 ; 0.75 ; 0.8\}$. The nominal significance level is $\alpha=5 \%$, and we report the rejection frequencies obtained from $M=10,000$ replications.

In Table 1 we see at the left hand side that the standard CUSUM test on differenced data (I) as well as the CUSUM-LM test (II) do not exceed their nominal size for all bandwidths and memory parameters and get close to $5 \%$ as the sample size increases. In most of the cases our approach is slightly more conservative.

On the right hand side of Table 1 the power results are given for a break size of one standard deviation in the middle of the sample. The power loss of our test is moderate especially at large bandwidths. The power of both tests increases as the bandwidth increases and it decreases as the memory parameter $d$ increases. The latter is due to the fact that means of long-memory processes converge only with a rate of $T^{1 / 2-d}$ instead of the usual rate of $\sqrt{T}$. Therefore, it is harder to identify changes of the mean in more persistent time series.

\section{Conclusion}

We conclude that our simple procedure delivers comparable results to the CUSUM-LM test for most financial time series where large samples are available. Furthermore, it benefits from the simplicity that it has a pivotal distribution which is independent of $\widehat{d}$. Hence, it is not necessary to simulate new critical values for every possible value of $\widehat{d}$ and CUSUM tests implemented in standard software can be used. 


\section{References}

Andreou, Elena and Eric Ghysels (2009). "Structural breaks in financial time series". In: Handbook of Financial Time Series. Springer, pp. 839-870.

Andrews, Donald WK (1991). "Heteroskedasticity and autocorrelation consistent covariance matrix estimation". In: Econometrica, pp. 817-858.

Brown, Robert L, James Durbin, and James M Evans (1975). "Techniques for testing the constancy of regression relationships over time". In: Journal of the Royal Statistical Society. Series B (Methodological), pp. 149-192.

Diebold, Francis X and Atsushi Inoue (2001). "Long memory and regime switching". In: Journal of Econometrics 105(1), pp. 131-159.

Granger, Clive WJ and Namwon Hyung (2004). "Occasional structural breaks and long memory with an application to the S\&P 500 absolute stock returns". In: Journal of Empirical Finance 11(3), pp. 399-421.

Horváth, Lajos and Piotr Kokoszka (1997). "The effect of long-range dependence on change-point estimators". In: Journal of Statistical Planning and Inference 64(1), pp. 57-81.

Krämer, Walter, Werner Ploberger, and Raimund Alt (1988). "Testing for structural change in dynamic models". In: Econometrica: Journal of the Econometric Society, pp. 1355-1369.

Krämer, Walter and Philipp Sibbertsen (2002). "Testing for Structural Changes in the Presence of Long Memory". In: International Journal of Business and Economics 1(3), pp. 235-242.

Künsch, H. R. (1987). "Statistical aspects of self-similar processes". In: Proceedings of the first world congress of the Bernoulli Society 1, pp. 67-74.

Marinucci, Domenico and Peter M Robinson (1999). "Alternative forms of fractional Brownian motion". In: Journal of Statistical Planning and Inference 80(1), pp. 111-122.

Newey, Whitney K and Kenneth D West (1987). "A simple, positive semi-definite, heteroskedasticity and autocorrelationconsistent covariance matrix". In: Econometrica 55(3), pp. 703-708.

Perron, Pierre et al. (2006). "Dealing with structural breaks". In: Palgrave Handbook of Econometrics 1, pp. 278-352.

Robinson, Peter M (1995). "Gaussian semiparametric estimation of long range dependence". In: The Annals of Statistics, pp. 1630-1661.

Wang, Lihong (2008). "Change-in-mean problem for long memory time series models with applications". In: Journal of Statistical Computation and Simulation 78(7), pp. 653-668.

Wright, Jonathan H (1998). "Testing for a Structural Break at Unknown Date with Long-memory Disturbances". In: Journal of Time Series Analysis 19(3), pp. 369-376. 